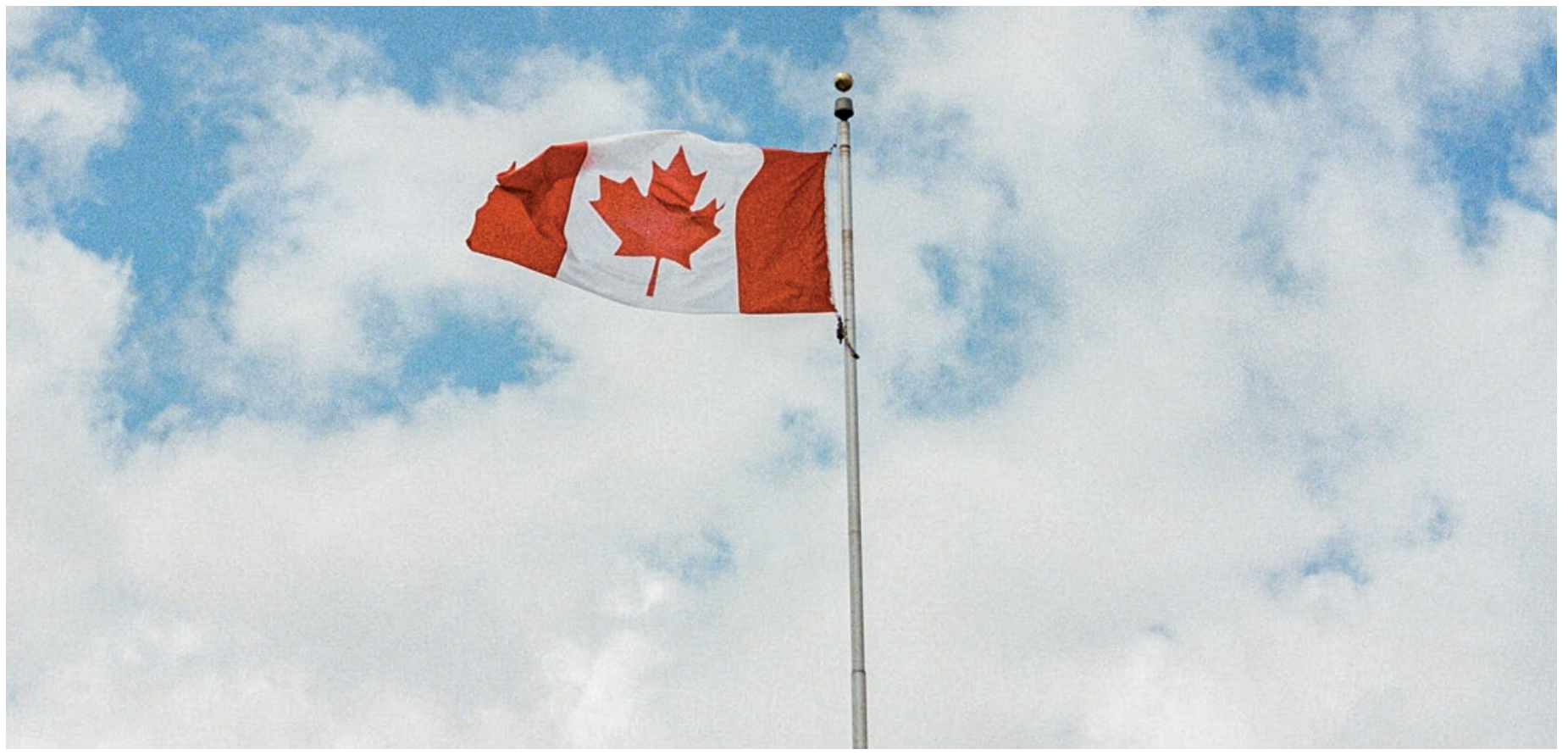

\title{
Interprofessionelle Ausbildung und kooperative Praxis in Kanada
}

\section{Marie-Andrée Girarda und John H. V. Gilbert ${ }^{\mathrm{b}}$}

a Doktorandin der Rechtswissenschaften, Universität Montréal, Adjoint-Professorin für Klinische Medizin, Medizinische Fakultät der Universität Montréal; b Professor emeritus, Universität von British Columbia, Senior Scholar, WHO Collaborating Centre on Health Workforce Planning \& Research, Dalhousie University, Ausserordentlicher Professor, Technische Universität Sydney, Gründungsvorsitzender der Canadian Interprofessional Health Collaborative

In Kanada ist die Interprofessionalität im Gesundheitswesen bereits seit rund 30 Jahren gelebter Alltag, während sie in der Schweiz erst langsam Fuss fasst. Wie funktioniert das kanadische Modell? Worauf beruht sein Erfolg? Einblicke in ein bewährtes, aber komplexes System, das auf Provinzen und zwei Amtssprachen aufgeteilt ist - Besonderheiten, die an die Schweiz erinnern.

Die interprofessionelle Ausbildung für kollaborative, patientenzentrierte Praxis und Pflege (Interprofessional education for collaborative person-centered practice and care, kurz: IPECP, im Folgenden als interprofessionelle Ausbildung bezeichnet) findet in einem komplexen Umfeld statt, in dem Fachkräfte des Gesundheitsund Sozialwesens und andere Interessengruppen daran arbeiten, eine sichere und hochwertige Pflege zu gewährleisten. In Kanada gibt es drei Hauptgründe für diese Komplexität.

Erstens sind die Gesundheits- und Sozialsysteme in Kanada und die Gesetzgebung, die sich auf sie bezieht, auf Provinzebene angesiedelt - auch wenn sie aufgrund der im Canada Health Act [1] enthaltenen Rechtsvorschriften teilweise von der Bundesregierung finan- ziert werden. Das kanadische Gesundheits- und Sozialsystem setzt sich also aus 13 verschiedenen Systemen zusammen, die völlig autonom sind. Dennoch gibt es eine föderale Standardisierung. Aufgrund der im Canada Health Act verankerten Finanzierungskriterien besteht eine gewisse Harmonisierung in Bezug auf den freien (öffentlichen) Zugang zu medizinisch notwendiger Versorgung und zu Einrichtungen der Akutversorgung, unabhängig von der Krankheit oder der Zahlungsfähigkeit einer Person. Darüber hinaus müssen die Akteure im Gesundheitswesen aufgrund des kanadischen Freihandelsabkommens [2] die Mobilität zwischen den Provinzen sicherstellen.

Zweitens hat Kanada zwei Amtssprachen. Eine Provinz ist offiziell französischsprachig, eine Provinz offiziell 
zweisprachig, acht Provinzen und drei Territorien sind englischsprachig. Deshalb erfordern pankanadische Harmonisierungsinitiativen ständige Übersetzungen in die beiden Amtssprachen.

Drittens liegen in Kanada die Verantwortung für das Bildungswesen einschliesslich der Hochschulbildung sowie die Verantwortung für die Regulierung der Berufe bei den Provinzbehörden. Der Zugang zur Berufsausbildung im Gesundheits- und Sozialwesen, die Verfügbarkeit solcher Programme und die Regulierung der Berufe variieren daher von Provinz zu Provinz und Territorium. Zudem haben die Provinzen und Territorien das Recht bzw. die Pflicht, ihre historische Sprache zu schützen.

Systemische Veränderungen in den Bildungs- und Gesundheits-/Sozialsystemen werden durch regulatorische und gesetzgeberische Fragen sowohl auf der Provinz- als auch der Bundesebene erschwert. Trotz dieser Schwierigkeiten ist die interprofessionelle Ausbildung in Kanada im Vergleich zu den 1960er Jahren weit fortgeschritten [3].

Maria Driedger (31), Hebammenstudentin in Hamilton, Ontario, Kanada

\section{"Andere Berufe sollen die Kompeten- zen der Hebammen kennen»}

"Bei uns in Kanada muss sich jede Frau am Anfang ihrer Schwangerschaft entscheiden, ob sie eine Hebamme oder eine Ärztin beziehungsweise einen Arzt für die Schwangerschaftsvorsorge und die Geburt haben möchte. Eine Kombination ist nicht möglich. Entscheidet sie sich für die Hebamme, führt diese alle Untersuchungen während der Schwangerschaft durch. Auch während der Geburt im Spital

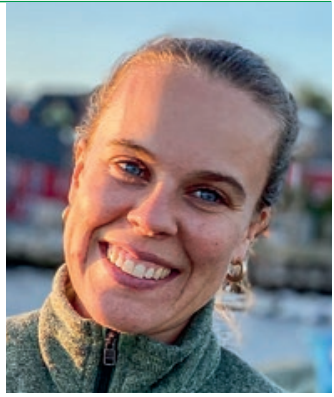
sind dann nur Hebammen dabei. Das alles natürlich unter der Voraussetzung, dass die Schwangerschaft und die Geburt unkompliziert verlaufen. Entscheidet sich die Schwangere aber für einen Arzt oder eine Ärztin, dann ist während der Geburt keine Hebamme dabei, und sie erhält keine Betreuung durch eine Hebamme nach der Geburt.

Diese Trennung hängt damit zusammen, dass der Hebammenberuf in Kanada in der ersten Hälfte des 20. Jahrhunderts abgeschafft worden ist, weil immer mehr Frauen ihre Kinder im Spital anstatt zu Hause bekamen. Erst während der Frauenbewegung ab den 70er Jahren setzten sich Aktivistinnen dafür ein, dass es wieder Hebammen geben sollte.

Ich mache derzeit einen 'Bachelor of Health Science in Midwifery': So heisst das Studium zur Hebamme an meiner Uni in Hamilton, das vier Jahre lang dauert. Im Moment bin ich im dritten Studienjahr, in dem ich interprofessionelle Praktika absolviere: Ich begleite zum Beispiel Pflegende, Stillberaterinnen und werde bald auch die Arbeit einer Gynäkologin oder eines Gynäkologen kennenlernen. Diese Praktika sind nicht nur für mich wichtig. Sie sind auch Teil des Studiums, damit die anderen Berufsgruppen besser verstehen, welche Kompetenzen wir Hebammen haben.

Die meisten Frauen gelangen während der Schwangerschaft nicht über ihre Arztpraxis an eine Hebamme, sondern über die Empfehlung anderer Frauen. Ärztinnen und Ärzte haben oft kein Interesse, unkomplizierte Fälle an uns Hebammen abzugeben. Denn dann würden sie nichts an der Geburt verdienen. Zwar gibt es in Kanada nicht genügend Hebammen für alle Schwangeren, aber wenn einmal ein Arzt oder eine Ärztin einer Frau ein hebammengeführtes Geburtshaus empfiehlt, versuchen die Hebammen oft, die Frau anzunehmen. Denn solch eine Empfehlung ist ein grosser Schritt in der interprofessionellen Zusammenarbeit und ein gutes Zeichen, dass wir beachtet und anerkannt werden.»

\section{Interprofessionelle Ausbildung heute}

Seit 1969 haben eine Reihe von Organisationen in ganz Kanada irgendeine Form von interprofessioneller Ausbildung eingeführt [3, 4]. Die Umsetzung war jedoch uneinheitlich. Aufgrund spezifischer Zulassungskriterien für die interprofessionelle Ausbildung, insbesondere für berufliche Studiengänge, wurde die interprofessionelle Ausbildung hauptsächlich vom akademischen Sektor vorangetrieben [5-7]. Gegenwärtig wird die Entwicklung der interprofessionellen kollaborativen Praxis in uneinheitlicher Weise durch berufs- oder verwaltungspolitische Massnahmen unterstützt [8, 9], obwohl sie seit Anfang 2000 als Eckpfeiler eines widerstandsfähigen öffentlichen Gesundheits- und Sozialsystems anerkannt ist [10]. Nach der Einführung von Zulassungskriterien in den entsprechenden Studiengängen haben viele Berufsregulierungsbehörden und ihre Disziplinargerichte den Begriff der kollaborativen Praxis in ihren Ethikkodex aufgenommen und damit begonnen, Verhaltensweisen zu sanktionieren, die die Teamarbeit behindern [11-13]. Einige Provinzregierungen haben die Gesetzgebungsstruktur ihrer Berufe geändert, um sich von strengen Berufssilos zu lösen und Gesetze und Verordnungen erlassen zu können, die die interprofessionelle Ausbildung erleichtern [12, 14, 15]. Mit den neuen Vorschriften werden Aufgabenverschiebungen und Aufgabenteilung möglich, was dem Ideal der geteilten Kompetenz und des sich überschneidenden Praxisbereichs entspricht $[12,13]$.

\section{Eine wachsende Praxis}

Unsere Organisation Canadian Interprofessional Health Collaborative (CIHC) hat ein Netzwerk von Lehrenden und Forschenden aufgebaut, die an der interprofessionellen Ausbildung in Kanada interessiert sind. Um sich ein Bild vom aktuellen Stand dieser Ausbildung im Land zu machen, führte die CIHC im Jahr 2020 eine Situationsanalyse einer Reihe von akademischen Programmen im ganzen Land durch.

Es wurde festgestellt, dass sich die interprofessionelle Ausbildung in Kanada weiterentwickelt. Die Zahl der Forschungspublikationen nimmt zu, und in den traditionellen Gesundheits- und Sozialberufen wird die Methode zunehmend einbezogen. Da die Bildungswege und Lehreinrichtungen recht unterschiedlich sind, überrascht es nicht, dass auch die Organisationsstrukturen und die Lehrpläne unterschiedlich sind. Sie reichen von Einrichtungen, in denen die interprofessionelle Ausbildung in das Längsschnittcurriculum nur eines Berufs eingebettet ist, ohne dass dafür eigene Ressourcen bereitgestellt werden, bis hin zu Einrich- 
tungen, in denen es ein offizielles Büro für die interprofessionelle Ausbildung gibt. Eine frühere Umfrage (2010) zeigte einen institutionellen Eigenverantwortungsprozess bei der Umsetzung von interprofessionellen Ausbildungsprogrammen, wobei der Prozess dem besonderen Umfeld jeder Institution Rechnung trug (CIHC-Situationsanalysebericht, unveröffentlichte Daten) [3].

Da die Mehrheit der Befragten in der Situationsanalyse 2020 von der Existenz eines interprofessionellen Ausbildungsprogramms berichtete, kann man mit Sicherheit sagen, dass die Zahl der Fachkräfte im Gesundheitswesen, die für eine kollaborative, patientenzentrierte Praxis ausgebildet sind, steigt. Die Interprofessionalität ist nun Teil der allgemeinen Lehrpläne von Gesundheitsfachkräften in ganz Kanada; dies ist möglicherweise auf die Einführung von spezifischen Zulassungskriterien für die interprofessionelle Ausbildung in vielen Berufen zurückzuführen $[7,16]$.

Die Mehrheit der Befragten gab an, dass ihre Dozenten und Studenten aus den Bereichen Krankenpflege, Medizin, Ergotherapie und Physiotherapie kommen, obwohl mehr als 25 Berufe und Tätigkeiten in interpro-

Hermann Dueck (65), ausgebildeter Spitalseelsorger, arbeitet in einem Altenheim in Winnipeg, Manitoba, Kanada, und macht Bereitschaftsdienste im Spital

\section{"Wir Seelsorgende tragen zur ganz- heitlichen Gesundheit bei»}

"In Kanada wird Gesundheit als das Wohlergehen von Leib, Seele und Geist definiert. Ausgebildete Spitalseelsorgende wie ich kümmern sich um die emotionale und geistliche Verfassung der Patientinnen und Patienten und nehmen als Experten für dieseThemen auch an interprofessionellen Fallbesprechungen teil. Die Pflegenden werden ermutigt, unseren Dienst zu empfehlen, aber natürlich handelt es sich um ein freiwilliges Angebot.

Nach einem Gespräch mit einer Patientin oder einem Patienten hinterlassen wir Rückmeldungen in der Krankenakte, damit Ärztinnen, Ärzte und Pflegende erfahren, wie das Gespräch lief und in welcher emotionalen Verfassung die Person ist. Nehmen wir an, ich spreche mit einem Ehepaar, dessen Kind kurz nach der Geburt gestorben ist. Dann beschreibe ich das Gespräch möglichst sachlich, ohne dabei das Seelsorgegeheimnis zu verletzen. Zum Beispiel so: 'Das Ehepaar war zutiefst erschüttert, ich habe sie ermutigt, ihre Erfahrung und ihre Gefühle mit mir zu teilen. Ich habe mich als empathischen Zuhörer angeboten, habe 30 Minuten mit ihnen verbracht, und meine Anwesenheit wurde sehr geschätzt. Ein weiteres Gespräch ist geplant.'

Solche Informationen sind wichtig, damit die behandelnden Personen das allgemeine Befinden der Patientinnen und Patienten berücksichtigen können. Die meisten Ärztinnen und Ärzte schätzen unsere Arbeit sehr, aber natürlich gibt es immer mal welche, die uns höchstens tolerieren. Zwei Beispiele dazu: Ein Arzt auf einer Palliativstation hat einmal eine längere Zeit vor dem Patientenzimmer gewartet, bis ich herausgekommen bin. Er wollte uns nicht stören, weil er meine Aufgabe in dem Moment als wichtiger ansah als seine. Ein anderer Arzt auf einer Intensivstation sagte mir einmal, man müsse dem Patienten mit der Überdosis an Drogen nur die richtigen Medikamente geben, dann werde alles wieder gut. Da habe ich vorsichtig widersprochen, denn echte Heilung ist nicht in jedem Fall nur mit Medikamenten möglich. Wir Menschen sind nicht nur ein Konglomerat von Organen, die gut funktionieren müssen, damit es uns gut geht. Deshalb tragen wir Seelsorgende zur ganzheitlichen Gesundheit bei.» fessionellen Ausbildungsprogrammen vertreten sind (CIHC-Situationsanalysebericht, unveröffentlichte Daten). Seit den AIPHE-Projekten (Accreditation of Interprofessional Health Education) Anfang der 2000er Jahre haben mehr als die acht Berufe, die sich in der ersten Phase der Akkreditierung angeschlossen hatten, Zulassungskriterien für die interprofessionelle Ausbildung eingeführt. Interessant ist, dass diese Programme die interprofessionellen Ausbildungskonzepte selbst in die Bildungsprogramme implementieren: Dies zeigt eine erfolgreiche Aufwertung und Integration der interprofessionellen Ausbildung [7].

Zunehmend sind auch die Regulierungsbehörden und die Gesundheitsministerien an der Entwicklung der interprofessionellen Ausbildung beteiligt. Sie haben begonnen, die Ideen der kooperativen Ausbildung und Praxis in ihren internen Richtlinien bzw. Ethikkodizes umzusetzen oder Schritte zu unternehmen, um die interprofessionelle Ausbildung zu erleichtern und rechtliche Hindernisse zu minimieren [8, 17-19].

\section{Welche Ziele es noch gibt}

In ihrem Strategieplan (2018) hat die CIHC ihre Vision für 2024 dargelegt. Darin heisst es, dass die CIHC eine starke, kohärente Stimme sein wird, um die nationale und globale Diskussion darüber zu verstärken und zu beeinflussen, dass interprofessionelle kollaborative Praktiken ein wesentliches Element des Gesundheitsmanagements, der Stärke und Widerstandsfähigkeit der Belegschaft, guter Behandlungsergebnisse und finanzieller Rechenschaft sind. Die CIHC hat sich zwei Hauptziele gesetzt:

a) bis 2024 als Anlaufstelle für die besten und vielversprechendsten Praktiken zu gelten, um zu verstehen, wie die interprofessionelle Zusammenarbeit in der Gesundheits- und Sozialpolitik, -praxis und -bildung wirklich funktioniert, und

b) bis 2024 als herausragende Quelle zu gelten, auf die man sich stützen kann, um die Entwicklung regionaler und lokaler Programme für die interprofessionelle Ausbildung zu beeinflussen.

Die CIHC sieht die dringende Notwendigkeit, die Stimme der Praktizierenden in ihre Arbeit einzubeziehen und sich auch mit dem Patient Voices Network zu vernetzen. Die CIHC erkennt zudem die Notwendigkeit, ihr Mitgliedschaftsmodell zu verändern, um eine solide finanzielle Basis ihrer Arbeit zu sichern. Zudem sollen Studierende als Fürsprecher für die interprofessionelle Ausbildung stärker eingebunden werden.

Derzeit ist die CIHC das einzige Sprachrohr für interprofessionelles Wissen, Ausbildung und Praxis in Ka- 
nada. Um ihren Auftrag zu erfüllen, verfolgt die CIHC unter anderem folgende Ziele:

1. Förderung der Nachhaltigkeit von interprofessionellen Ausbildungsprogrammen an Universitäten, Fachhochschulen und Instituten in ganz Kanada durch:

a) Gewährleistung regelmässiger finanzieller Unterstützung, Tenure-Track-Förderung und kontinuierlicher Forschung,

b) Integration der Fähigkeit zur Zusammenarbeit in die Führung im Gesundheits- und Sozialwesen,

c) Sicherstellung der Bedeutung der interprofessionellen Ausbildungs-Akkreditierungsstandards im Hinblick auf den kulturellen Wandel in der Ausbildung von Fachkräften des Gesundheitsund Sozialwesens;

2. Förderung und Stärkung von Strategien für die interprofessionelle Ausbildung und Minimierung rechtlicher und politischer Hindernisse für die effektive interprofessionelle Ausbildung;

3. Förderung der interprofessionellen Ausbildung als Instrument zur Lösung der grossen Probleme beim Zugang zu Fachkräften im Gesundheits- und Sozialwesen in ländlichen und abgelegenen $\mathrm{Ge}$ meinden;

4. Förderung der interprofessionellen Ausbildung als Grundlage für die Stärke und Widerstandsfähigkeit der Arbeitskräfte;

Vollständige Literaturliste unter www.saez.ch oder via QR-Code

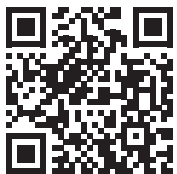

John H. V. Gilbert johnhvg[at]mail.ubc.ca

Marie-Andrée Girard marie-andree.girard.1[at] umontreal.ca
5. Durchführung von Situationsanalysen zum Stand und Status der interprofessionellen Ausbildung in Kanada und zum Nachweis bewährter Verfahren.

\section{Das kanadische Modell: weltweit bekannt}

Obwohl die Konzepte der interprofessionellen Ausbildung 1964 an der University of British Columbia in Kanada entwickelt wurden, war es die Veröffentlichung der Royal Commission on the Future of Health Care in Canada (auch bekannt als Romanow-Bericht) im November 2002, die weitreichende Änderungen empfahl, um die langfristige Nachhaltigkeit des kanadischen Gesundheitssystems zu gewährleisten. Jener Bericht führte dazu, dass die interprofessionelle Ausbildung als ein Grundsatz des kanadischen Gesundheits- und Sozialsystems anerkannt wurde. Im Anschluss finanzierte Health Canada vier Jahre lang interprofessionelle Ausbildungsprojekte in ganz Kanada, wobei eine Reihe von akademischen und klinischen interprofessionellen Ausbildungsprogrammen eingerichtet wurden, die die Grundlage für ein System bildeten, das noch immer in Kraft ist.

Die Arbeit der von Health Canada finanzierten Projekte umfasste den CIHC-Kompetenzrahmen und die Arbeit an der Akkreditierung (AIPHE), die weltweit bekannt sind. Die Entwicklung von Richtlinien und Verfahren führte auch zu zahlreichen Veröffentlichungen, die die interprofessionelle Ausbildung weltweit beeinflussten. Zudem war die Beziehung der CIHC mit der American Interprofessional Health Collaborative (AIHC) beim Aufbau der Konferenzreihe Collaborating Across Borders ein globaler Beweis für die Wirksamkeit grenzüberschreitender Initiativen in diesem Bereich und hat gleichzeitig die Teilnahme von Ländern ausserhalb Kanadas und der USA gefördert.

Kanada und die Schweiz stehen bei der Entwicklung der interprofessionellen Ausbildung für kollaborative, patientenzentrierte Praxis und Pflege über das gesamte Bildungs- und Betreuungskontinuum hinweg vor ähnlichen Herausforderungen. Die Erfahrungen Kanadas könnten für die Initiativen in der Schweiz von Nutzen sein.

Bildnachweis

Jp Valery / Unsplash

\section{Schwerpunktserie Interprofessionalität}

Die interprofessionelle Zusammenarbeit von Fachpersonen aus verschiedenen Gesundheitsberufen gilt als wichtiges Mittel, um den Herausforderungen im Gesundheitswesen zu begegnen. Aber wie weit ist die Schweiz in diesem Bereich tatsächlich? Welche Hürden und Chancen gibt es? In unserer Schwerpunktserie betrachten wir dasThema aus unterschiedlichen Perspektiven. 


\section{Literatur}

1 Loi Canadienne Sur La Santé.

2 LC 2017, c 33, art 219 | Loi de mise en œuvre de l'Accord de libre-échange canadien. Accessed November 30, 2021. https://www.canlii.org/fr/ca/ legis/lois/lc-2017-c-33-art-219/derniere/lc-2017-c-33-art-219.html?resultIndex=12

3 Gilbert J.H.V. The status of interprofessional education in Canada. J Allied Health. 2010;39(SUPPL. 1):216-23.

4 McCreary JF. The Education of Physicians in Canada. Can Med Assoc J. 1964;90(21):1215-21.

5 Accreditation of Interprofessional Health Education (AIPHE). Accreditation of interprofessional health education (AIPHE) principles and practices for integrating interprofessional education into the accreditation standards for six health professions in Canada. Retrieved from. Published online 2009. http://www.cihc.ca/aiphe/

6 Accreditation of Interprofessional Health Education (AIPHE). Interprofessional Health Education Accreditation Standards Guide Phase 2. Published online 2012. http://www.cihc.ca/files/resources/public/English/AIPHE\%2OInterprofessional\%2OHealth\%2OEducation\%20 Accreditation\%20Standards\%20Guide_EN.pdf

7 Azzam M, Puvirajah A, Girard MA, Grymonpre RE. Interprofessional education-relevant accreditation standards in Canada: a comparative document analysis. Human Resources for Health. 2021;19(1):66. doi:10.1186/s12960-021-00611-1

8 Girard MA, Régis C, Denis JL. Interprofessional collaboration and health policy: results from a Quebec mixed method legal research. Journal of Interprofessional Care. Published online May 6, 2021:1-8. doi:10.1080/13561820.2021.1891030

9 Suter E, Mallinson S, Misfeldt R, Boakye O, Nasmith L, Wong ST. Advancing team-based primary health care: a comparative analysis of policies in western Canada. BMC Health Services Research. 2017;17(1). doi:10.1186/s12913-017-2439-1

10 Romanow RJ, Commission sur l'avenir des soins de santé au Canada. Guidé par nos valeurs l'avenir des soins de santé au Canada: rapport final. Commission sur l'avenir des soins de santé au Canada; 2002. Accessed May 16, 2016. http://dsp-psd.communication.gc.ca/Collection/ CP32-85-2002F.pdf

11 Trudeau JB, de Grandmont S, Lafrance L, Poitras L. la «loi 90»: la force de l'interdisciplinarité. Des connaissances et des compétences à partager. In: Le Système Socio-Sanitaire Au Québec; Gouvernance, Régulation et Participation. Gaëtan Morin Editeur;2007:263-72.

12 Bélanger L, Roy C, Simard MC, Trudeau JB. Partager des activités professionnelles pour mieux collaborer. Service de la formation continue du Barreau du Québec, 2019. 2019; Développements récents en droit de la santé (2019) (EYB2019DEV2790).

13 Bourgeault IL, Grignon M. A comparison of the regulation of health professional boundaries across OECD countries. The European Journal of Comparative Economics. 2013;10(2):199-224.

14 Lahey W, Fierlbeck K. Legislating collaborative self-regulation in Canada: A comparative policy analysis. Journal of Interprofessional Care. 2016;30(2):211-6. doi:10.3109/13561820.2015.1109501

15 Lahey W. Legislating Interprofessional Regulatory Collaboration in Nova Scotia. Health Reform Observer-Observatoire des Réformes de Santé. 2013;1(1). doi:10.13162/hro-ors.01.01.04

16 Curran V. Environmental scan report: Interprofessional education and accreditation processes in pre-licensure health professional education Canadian Interprofessional Health Collaborative URL: http://www cihc ca/files/aiphe/resources/AIPHE\% 20Environmental\% 20Scan\% 20Report pdf. Published online 2008. Accessed March 21, 2016. http://www.cihc.ca/files/resources/public/English/AIPHE\%2OEnvironmental\%20Scan\%20 Report.pdf

17 Girard MA. Interprofessional education and collaborative practice policies and law: an international review and reflective questions. Human Resources for Health. 2021;19(1):9. doi:10.1186/s12960-020-00549-w

18 Girard MA. Interprofessional Collaborative Practice and Law: A Reflective Analysis of 14 Regulation Structures. Journal of Research in Interprofessional Practice and Education. 2019;9(2).

19 Ries NM. Law matters: How the legal context in Canada influences interprofessional collaboration. Journal of Interprofessional Care. 2017;31(4):417-9. doi:10.1080/13561820.2017.1310495 